\title{
Pressure field in measurement section of wind tunnel
}

\author{
Jakub Hnidka ${ }^{1, *}$ and Dalibor Rozehnal ${ }^{1}$ \\ ${ }^{1}$ University of Defence, Department of Air Force and Aircraft Technology, Kounicova 65, \\ 66210 Brno, Czech Republic
}

\begin{abstract}
The University of Defence in Brno has a new low-speed wind tunnel. In order to confirm the quality of the wind inside of the measurement section, several measurements of the dynamic pressure have been performed with the Pitot-static tube. The pressure fields are then analysed and quality of the field is evaluated. Measurement of a pressure drop on the body of a standing helicopter was conducted.
\end{abstract}

\section{Introduction}

A new low-speed wind tunnel of return-flow type with an open measurement section was built at the University of Defence. The dimensions of the measurement section (MS) are $830 \times 570 \times 1370 \mathrm{~mm}$ (width $\times$ height $\times$ length). The maximum velocity is currently $\mathrm{M}=0.09(30 \mathrm{~m} / \mathrm{s})$; nonetheless, the fan currently operates in a restricted mode and the velocity will be increased in the future. Due to the relatively low values of Reynolds number achieved, the wind tunnel is ideal for e.g. testing the flight performance of small UAVs, which can be measured in scale of 1:1.

Investigation of the flow characteristics of the wind tunnel is a necessary step in the optimization of the wind tunnel [1]. E.g. in propeller testing, the aerodynamic performance of the propeller can be expressed as its efficiency. At a constant rotational speed of the propeller, a $1 \%$ change of the freestream velocity in the plane of rotation near the propeller take-off design point can alter the measured efficiency of the propeller by $0.5 \%$ and power absorbed by propeller by $1.3 \%$ [2]. If the wind tunnel is to be used to measure flight performance of the small UAVs, which almost exclusively relay on the propeller as a source of thrust, non-uniform velocity along the freestream could significantly influence the measured results.

The wind tunnel is equipped with vanes in all corner sections of the tunnel. Two corners upstream from the nozzle feature water-cooled vanes. Temperature stability of the freestream in the MS was investigated as well.

The flow fluctuation will be investigated in the close future.

\footnotetext{
${ }^{*}$ Corresponding author: jakub.hnidka@unob.cz
} 


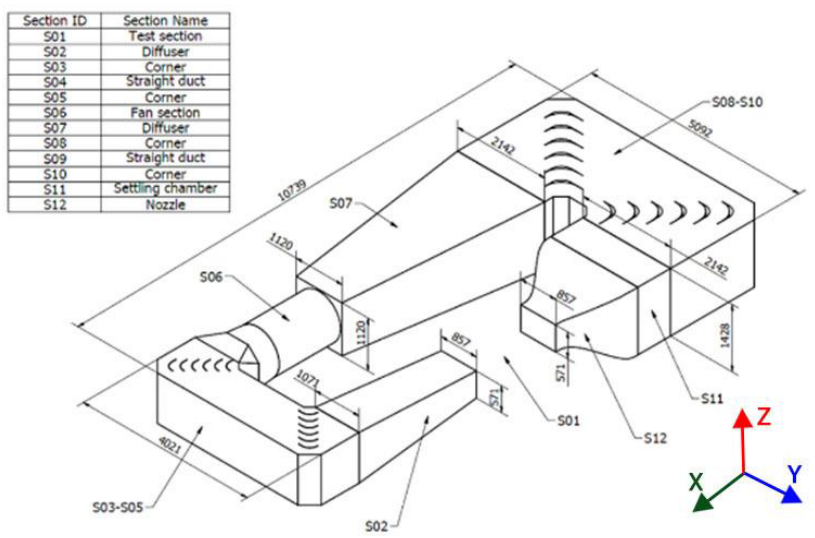

Fig. 1. Wind tunnel geometry with marked sections [3]. In the right bottom corner is shown the orientation of axes in the MS. $X$-axis is in the direction of wind flow.

\section{Measurement instrumentation}

The measurement of the velocity was performed with Pitot-static probe mounted on the traverse system. The system is capable of moving the probe in three axis to any destination in the MS. The system is controlled by three stepper motors, which are wired to the NI-7330 Motion Control PCI Module.

A differential pressure sensor with analogue output measured the dynamic pressure. The signal was sampled by NI 9215 module with 16-bit A/D converter, $100 \mathrm{kS} / \mathrm{s} / \mathrm{ch}$ maximum sampling speed and four BNC terminals [4]. This module was fitted to cDAQ-9184 chassis, which were connected to the PC via Ethernet bus. During the measurement, the application was testing the measurement capabilities of the system and it performed FFT analysis of the raw data. The purpose of this analysis was to search for any regular occurrences, which could indicate disruption of the freestream, sourced in e.g. screens, or blades of the fan. The differential pressure sensor was mounted outside of the traverse system in order to prevent any influence of the data by vibrations caused by the wind tunnel. The tubes connecting the sensor with the Pitot-static tube are therefore of a considerable length. Pressure measurement assumes the incompressibility of the air in the connecting tubes and this criterion is met for static events. Tested hypothesis was that if there is a significant disruption of the freestream, it could be measured by the pressure sensor despite the damping in the connecting tubes. Results of this analysis are not part of this paper - nonetheless, in order to observe higher frequencies, the sampling rate was set to $5 \mathrm{kHz}$. Occurrence of low frequencies was investigated separately, because the measurement of dynamic pressure distribution is time-consuming. The MS is divided into finite number of points in selected cross sections, where each node of the mesh is measured. The system implements a waiting procedure, where before initializing the measurement, it waits for a certain period of time (typically 1 second) and then starts the measurement. This procedure has a major impact on the overall duration of the measurement and because the wind tunnel is temperature sensitive device, the measurement time was reduced to acceptable minimum of 2 seconds. Stationarity of the freestream was evaluated separately, where the probe performed measurement 
in few selected nodes with measurement time up to 120 seconds. Average values of dynamic pressure measured in both cases are well comparable and measurement time of 2 seconds is long enough to correctly estimate the dynamic pressure in the measured point.

During the measurement, the ambient pressure and temperature of the freestream were measured separately. In total, six RTD sensors Pt1000 were used to measure temperature in five different sections of the wind tunnel and ambient temperature. Two four-channel NI 9219 modules with 24-bit A/D converter [5] were used to sample resistance measured at the sensors. Time-synchronization had to be solved, because NI 9219 modules used the same sampling clock as NI 9215 (pressure). Reference temperature used to calculate the velocity was measured at the nozzle output.

An application was written in LabVIEW, which both controls the pressure and temperature measurement and the motion of the system [6].

Data were evaluated in LabVIEW with implementation of Advanced Plotting Toolkit [7].

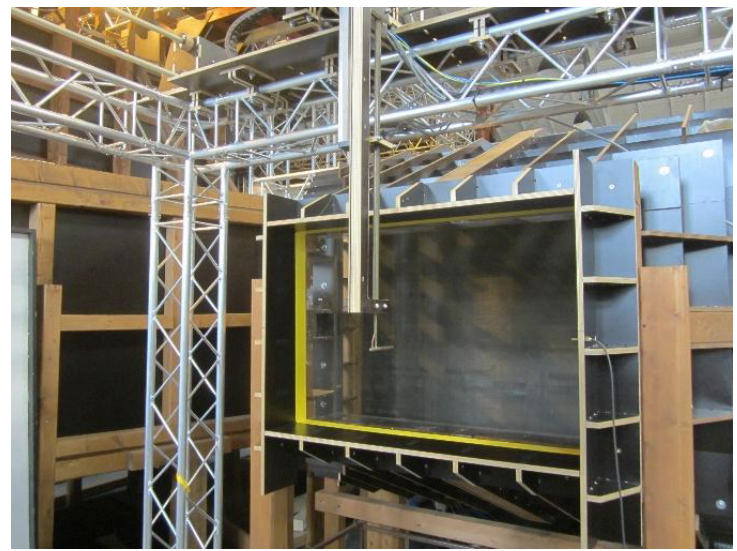

Fig. 2. Measurement of dynamic pressure distribution in $\mathrm{X}=0$.

\section{Dynamic pressure distribution measurement}

Dynamic pressure distribution along the MS is one of the key parameters of the wind tunnel. It is necessary to precisely map its changes with relation to the velocity achieved in the MS. Typically, the velocity in aerodynamics is expressed as a Mach number, or the ratio between the actual velocity $v$ and speed of sound $a$.

$$
M=\frac{v}{a}
$$

The flowfield investigation was conducted at Mach numbers of $0.030,0.045$, $0.060,0.075$ and $0.090(10,15,20,25,30 \mathrm{~m} / \mathrm{s})$ in four different cross sections of the MS, distanced from the nozzle output by $0,200,400$ and $600 \mathrm{~mm}$ downstream. The length of the MS is $1370 \mathrm{~mm}$ - the model is typically mounted $400 \mathrm{~mm}$ downstream from the nozzle output and the cross sections further downstream were not investigated yet. The results were also compared to a measurement conducted several years ago before the internal parts of the wind tunnel were tuned.

Measured data were evaluated in accordance with [2]. 


\subsection{Procedure of data evaluation}

Pitot-static probe measures total and static pressure - their difference is equal to dynamic pressure $q_{i}$. The velocity $v_{i}$ can be expressed:

$$
v_{i}=\sqrt{\frac{2 q_{i}}{\rho_{i}}}
$$

Due to the temperature instability, the air density $\rho$ changes during the measurement. The density is not measured directly, but as a function of the ambient pressure $p_{a m b}$. and temperature of the air in the MS $T_{i}$ :

$$
\rho_{i}=\frac{p_{a m b}}{r \cdot T_{i}}
$$

The specific gas constant of the air $r$ is equal to $287.1 \mathrm{Jkg}^{-1} \mathrm{~K}^{-1}$. The ambient pressure is measured separately and it is considered to be constant during the measurement.

Distribution of both velocity and dynamic pressure is calculated. The ratio of dynamic pressures is more severe criterion than velocity - the velocity is proportional to the square root of the dynamic pressure (2). The dynamic pressure distribution ratio $\bar{q}$ is calculated according to formula:

$$
\bar{q}=\frac{q_{i}}{q_{r e f}}
$$

The dynamic pressure $q_{i}$ is related to the reference pressure $q_{r e f}$, which is measured at the geometrical centre of the measured cross section area. Similarly, the velocity ratio $\bar{v}$ :

$$
\bar{v}=\frac{v_{i}}{v_{r e f}}
$$

The change of the dynamic pressure in the geometric centre of each cross section measured along the MS is calculated in accordance with (4).

\subsection{Temperature instability during the measurement}

The precision of the measurement is related to the temperature stability inside of the MS during the measurement. Based on the equations (2) and (5), 2\% change of the temperature can cause almost $1 \%$ change of the velocity measured.

During the measurement, the air inside of the wind tunnel is heated by friction until a certain equilibrium is reached. Due to relatively small Mach numbers reached, any significant change of the temperature could be avoided by accelerating the wind tunnel to higher velocity to pre-heat the air inside and then decelerating to the desired value of velocity. 


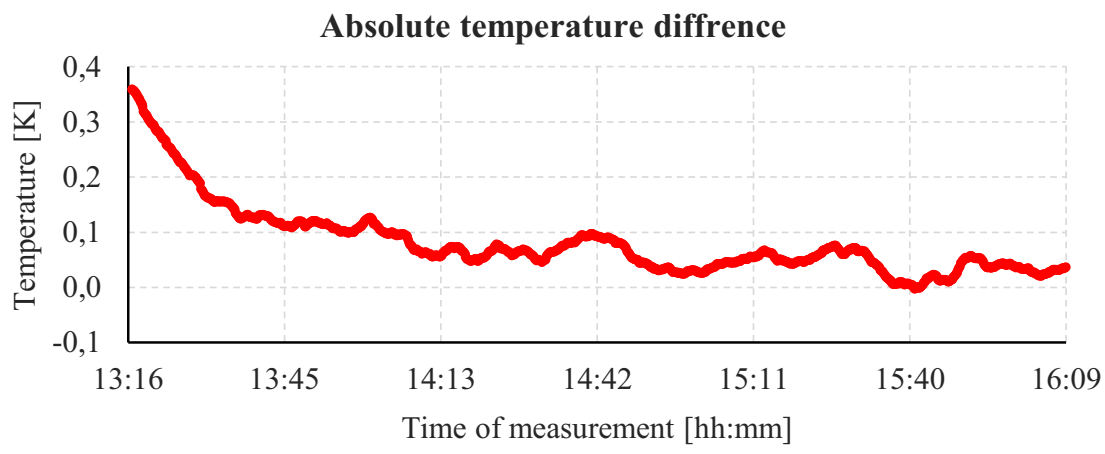

Fig. 3. Absolute temperature difference during the measurement at $M=0.03(10 \mathrm{~m} / \mathrm{s})$.

The absolute temperature difference during the measurement at the $\mathrm{M}=0.03$ is shown in the Fig. 3. The temperature difference significantly drops in the first phase of the measurement. The pre-heating process causes this phenomenon. The temperature is stable during the measurement.

The ambient temperature does change during the measurement and it can affect the measurement. It is crucial to perform any measurement in the wind tunnel during as stable ambient conditions, as possible. Fig. 4. shows record of temperature during three-hour long measurement around noon - the temperature inside of the wind tunnel Temp changes only by $0.2 \mathrm{~K}$. Tamb. is the ambient temperature.

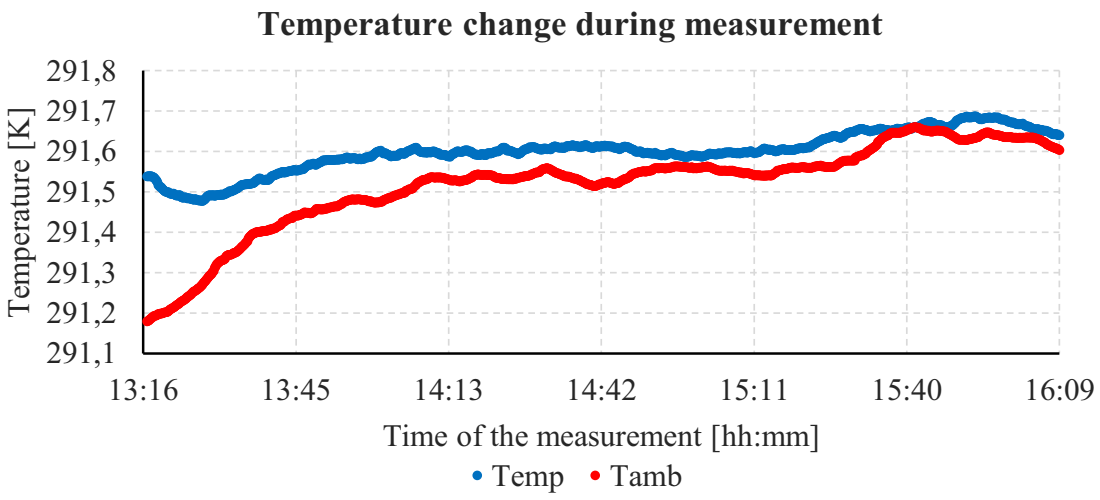

Fig. 4. Temperature change during a measurement at $M=0.03(10 \mathrm{~m} / \mathrm{s})$.

Measured velocity change is proportional to the square root of temperature change:

$$
\Delta v \sim \sqrt{\Delta T e m p}
$$

According to (6) in an example demonstrated in Fig. 4, temperature change from $291.5 \mathrm{~K}$ to $291.7 \mathrm{~K}$ requires $0.034 \%$ correction of the measured velocity. Temperature screening during the measurement is used to confirm the temperature stability in the MS and temperature corrections are used only if the temperature change is significant. 
The controller of the wind tunnel maintains a constant pre-set frequency of the fan. The temperature change is then inversely proportional to the pressure delivered by the fan. This phenomenon further increases the impact of the ambient temperature change to the precision of the measurement.

\subsection{Flowfield measurement results in the cross section of MS}

The investigation of the dynamic pressure and velocity distribution along the measurement section confirms uniform profile of the flowfield at all Mach numbers currently achievable in the wind tunnel. The Fig. 5-8 focus on the area, where the change of dynamic pressure is within $\pm 1 \%$ or change of velocity within $\pm 0.5 \%$.

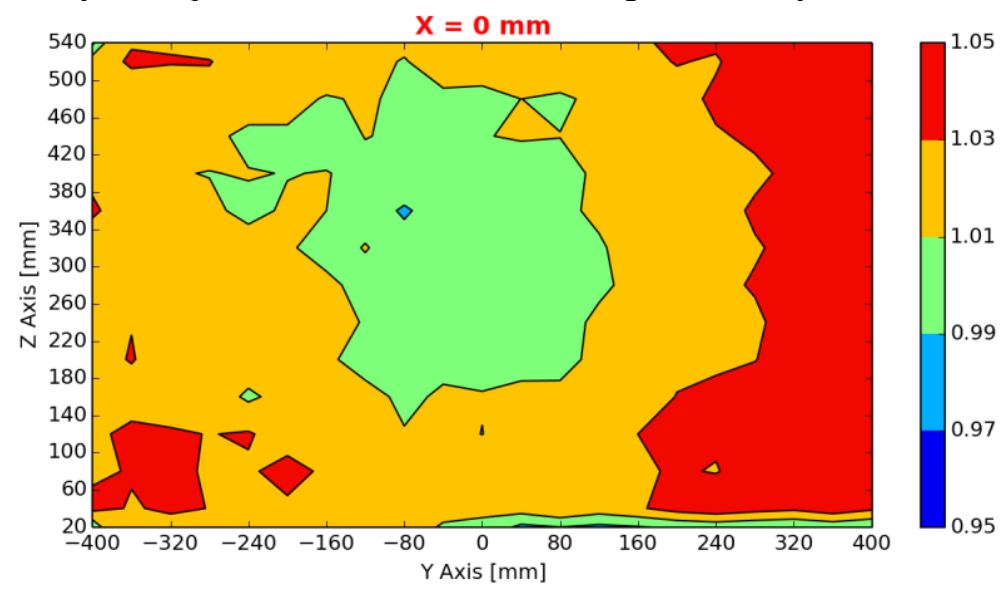

Fig. 5. Dynamic pressure at the output of the nozzle at $M=0.075(25 \mathrm{~m} / \mathrm{s})$.

Worst parameters were observed at the output of the nozzle, due to its significant contraction (more than 6:1) as shown in Fig. 5. There is a noticeably asymmetrical profile caused by the setup of the vanes in the corner of the wind tunnel upstream of the nozzle.

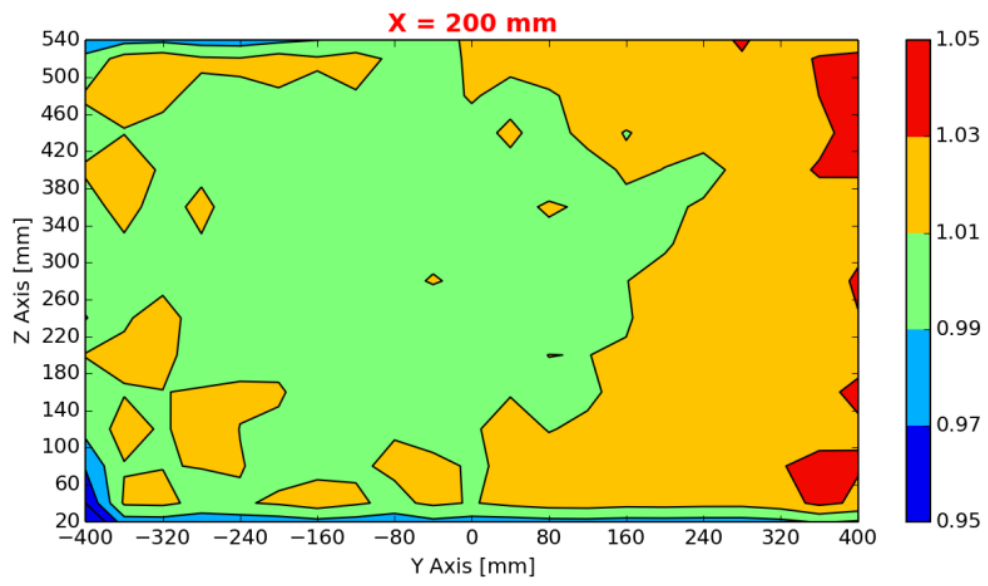

Fig. 6. The dynamic pressure distribution in the MS at $M=0.075(25 \mathrm{~m} / \mathrm{s})$. 
$200 \mathrm{~mm}$ downstream the dynamic pressure distribution is almost entirely within $\pm 1 \%$ change as shown in the Fig. 6 .

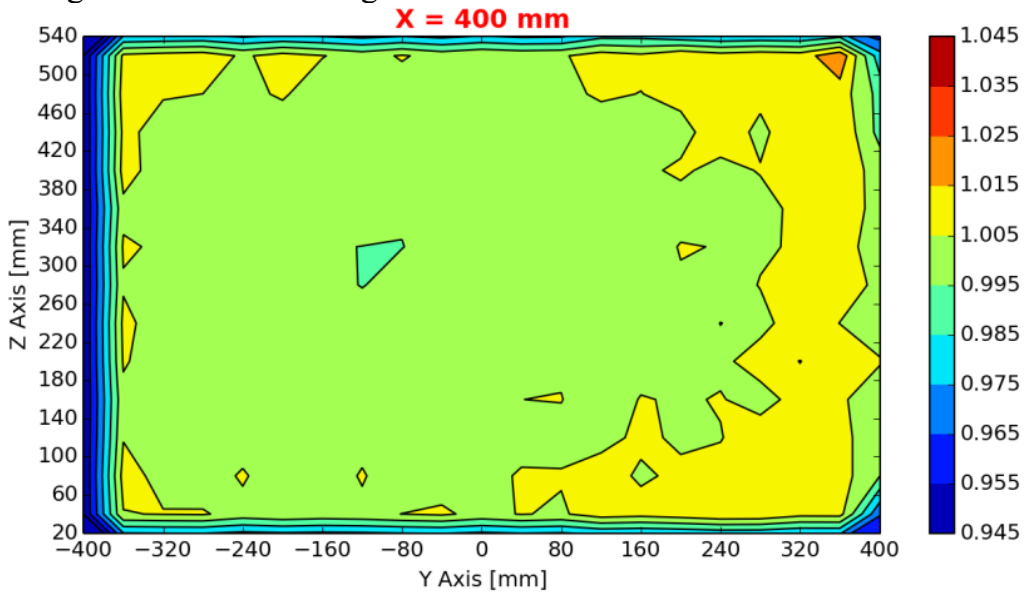

Fig. 7. Velocity distribution at $\mathrm{M}=0.075(25 \mathrm{~m} / \mathrm{s})$.

The flow in the open MS expands, which tends to smooth out any differences in the pressure and velocity. The $\mathrm{X}=400 \mathrm{~mm}$ is the cross section, where model is typically mounted. The velocity change in the cross section is mostly within a $\pm 0.5 \%$ of a change.

Fig. 8 confirms uniform velocity profile $600 \mathrm{~mm}$ downstream of the nozzle. The velocity distribution is within $\pm 0.5 \%$ of a change in the large area of the cross section.

Similar parameters were observed for all Mach numbers measured.

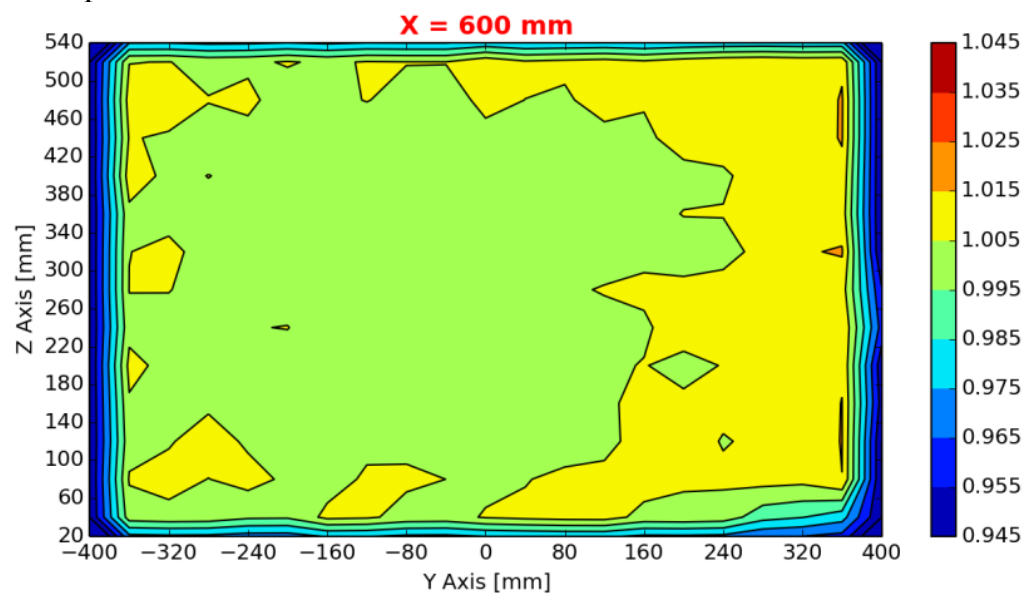

Fig. 8. Velocity distribution at $M=0.075(25 \mathrm{~m} / \mathrm{s})$.

\subsection{Reference pressure distribution along the MS}

The reference pressure/velocity was obtained as an average measured at the geometric centre of the measured cross section. This pressure is not constant in the downstream direction. 
The contraction of the nozzle is considerable, causing the stream to accelerate in the MS. However, at a certain distance, the freestream starts expanding, causing the pressure drop. This pressure drop is more significant at higher Mach numbers.

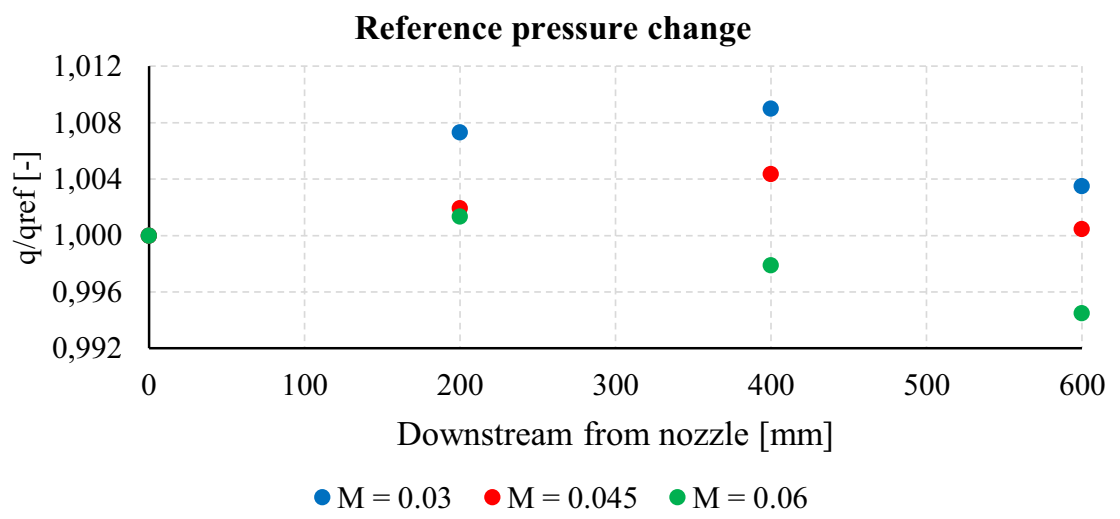

Fig. 9. Reference pressure change in the downstream direction in the MS.

\subsection{Comparison of a flowfield parameters in the MS}

During the development of the wind tunnel, numerous times were the flowfield parameters (i.e. dynamic pressure and corresponding freestream velocity) investigated - mostly to confirm the positive impact caused by any alteration caused to the wind tunnel configuration.

The very first measurement was performed manually - the traverse system was installed later. In addition, the wind tunnel was not properly configured. Yet, the measurement showed promising results, as shown at the left side of Fig. 10. The unnatural symmetry was achieved by mirroring the results, because the instrumentation at the time could not reach other side of the wind tunnel. In the right side of the Fig. 10 is the current state of the flowfield parameters.
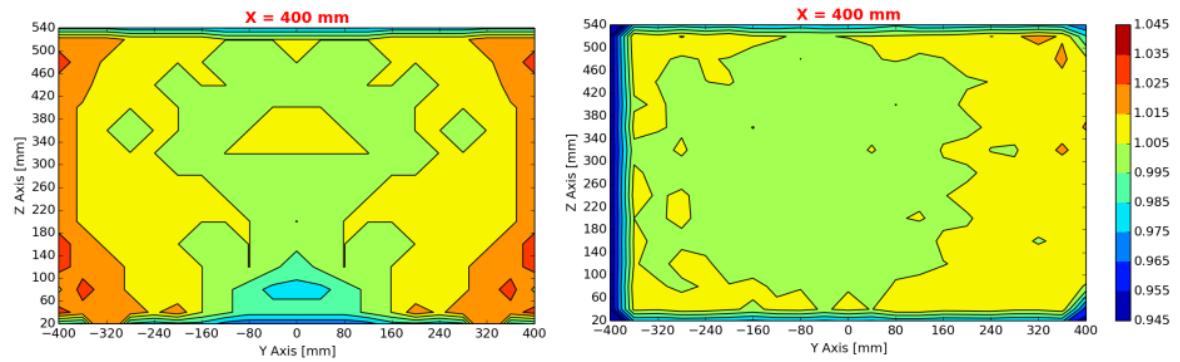

Fig. 10. Comparison of flowfield parameters in the MS along the time at $M=0.045$.

The symmetry in the original data was only assumed - the measurement instrumentation measured only right half of the MS and the results were extrapolated to the left half of the MS. The alterations to the inside of the wind tunnel - among others described in [3] - considerably improved the flowfield parameters of the wind tunnel. 


\section{Measurement of the dynamic pressure loss on the model of a helicopter}

The traverse system can be used not only to map the velocity profiles in the empty MS, but it can also be used to investigate the pressure drop on a model mounted in the MS.

If the flowfield parameters are constant, then the drop of dynamic pressure between cross section upstream and downstream from the model is proportional to its drag. Therefore, it is not necessary to implement a balance system to measure the drag directly. However, typically a balance system is more preferable, because as such, it is a much less time-consuming method.

Measurement of a dynamic pressure drop was performed at the model of the Mi24 helicopter in order to investigate the dynamic pressure downstream from the model. Purpose of this measurement was to test the traverse system and its ability to map dynamic pressure behind standing aircraft, which is subjected to a significant cross- or headwind. The pressure loss was determined by measurement of the dynamic pressure in the cross section upstream and downstream from the model - the cross sections were as close to the model as possible. The pressure drop $q(\%)$ was then evaluated as difference of dynamic pressure upstream $q(u p s, y, z)$ from the model and dynamic pressure downstream $q(d w s, y, z)$ from the model - in each node with the same coordinates $(y, z)$.

$$
q(\%)=\frac{q(d w s, y, z)-q(u p s, y, z)}{q(u p s, y, z)} 100 \%
$$

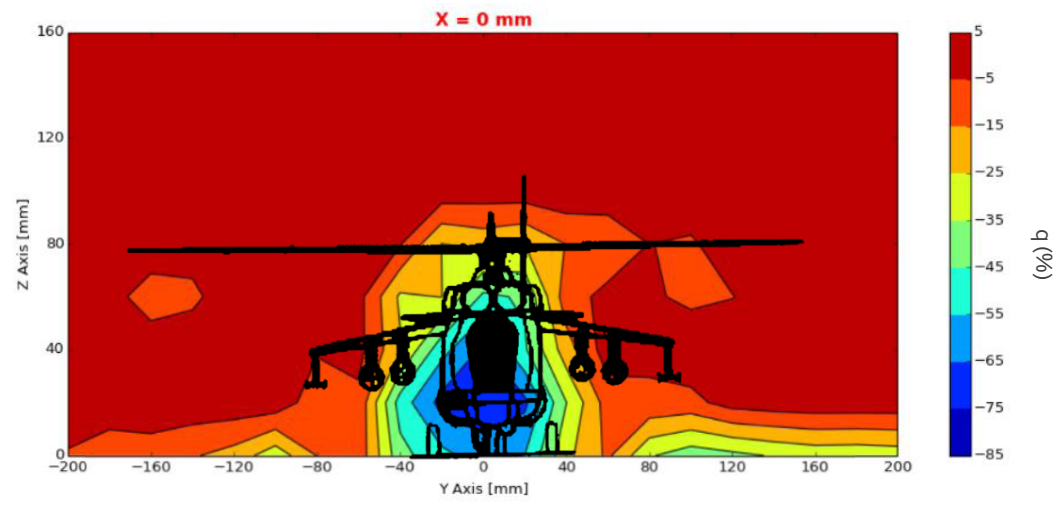

Fig. 11. Dynamic pressure change in the cross section downstream from a model at $M=0.06$ $(20 \mathrm{~m} / \mathrm{s})$.

The results of measurement of headwind are shown in the Fig. 11. The pressure drop was significant and the pressure field marks the silhouette of the model of the helicopter. The traverse system mapped precisely the dynamic pressure. 


\section{Conclusion}

The investigation of flowfield parameters (i.e. dynamic pressure and velocity) in MS of the wind tunnel proved that the velocity distribution along the MS is uniform. With exception of the nozzle output, where there are still prevailing distortions caused by a considerable contraction of the nozzle, the velocity ratio is in the most parts of the MS within $\pm 1 \%$ for all Mach numbers measured up to $M=0.09(30 \mathrm{~m} / \mathrm{s})$.

The wind tunnel currently is not featuring a heat exchanger and the temperature inside of the MS cannot be controlled. Known issue with a return-flow type wind tunnel is the heat surplus caused by the friction. Temperature changes are monitored and temperature increase was investigated. Due to the relatively low Mach numbers achieved, the temperature change during the measurement can be minimized by preheating the wind tunnel by accelerating to higher velocity then desired and then decelerating. Because of the considerable duration of the measurement, a correlation between ambient temperature and temperature in the wind tunnel can be observed.

The wind tunnel delivers a uniform air flow in the MS and as such is ideal e.g. for measurements of a small UAV, where the velocity achieved is not large and as such typically operate at very low Reynolds numbers. The wind tunnel was used also to measure the impact of the crosswind and headwind to the standing aircraft. In this case, a model of a Mi-24 was used and a pressure drop was recorded.

This work was completed as a result of a cooperation with the company ENERGOKLASTR.

\section{References}

1. J. B.Barlow, W. H. Rae and A. Pope. Low-speed wind tunnel testing (3rd ed. New York: Wiley, c1999)

2. CH. Hughes, Flowfield Measurements in the NASA Lewis Research Center 9- by 15-Foot Low-Speed Wind Tunnel: NASA Technical Memorandum 100883 [online]. (Lewis Research Center Cleveland, Ohio, 1989) [quot. 2017-03-05]. Available from: https://ntrs.nasa.gov/archive/nasa/casi.ntrs.nasa.gov/19890011631.pdf

3. V. Havránek, CFD Analysis of Wind Tunnel Components [quot. 2017-03-05]. (Diploma thesis. University of Defence. Supervisor doc. Ing. Dalibor Rozehnal, Ph.D. Brno, 2015)

4. NI 9215 Datasheet [online]. National Instruments, 2016 [quot. 2017-03-05]. Available from: http://www.ni.com/pdf/manuals/373779a_02.pdf

5. NI 9219 Datasheet [online]. National Instruments, 2014 [quot. 2017-03-05]. Available from: http://www.ni.com/datasheet/pdf/en/ds-198

6. J. Hnidka, Design of traverse 3D system for low-speed wind tunnel (Diploma thesis. University of Defence. Supervisor Doc. Ing. Dalibor Rozehnal, Ph.D. Brno, 2015)

7. Advanced Plotting Toolkit [online]. National Instruments [quot. 2017-03-05]. Available from: http://sine.ni.com/nips/cds/view/p/lang/cs/nid/213166 